\title{
Dysmenorrhea in Young People: Experiences from a Tertiary Centre with a focus on
}

\section{Conservative Management}

Aalia Sachedina ${ }^{1}$, Mardiha Abu Bakar², Angela M Dunford ${ }^{3}$, Amanda Morris $^{4}$, Abdul Ghani Nur Azurah $^{5}$, Sonia R Grover ${ }^{1,6,7}$

1. Department of Gynaecology, The Royal Children's Hospital, Victoria, Australia

2. General Practice, Creswick and Clunes Medical Centre, Victoria, Australia

3. Department of Gynaecology and Women's Health, John Hunter Hospital, New South Wales, Australia

4. University of Manitoba, Manitoba, Canada

5. Department of Obstetrics \& Gynaecology, Universiti Kebangsaan Malaysia, Kuala Lumpur, Malaysia

6. Department of Paediatrics, University of Melbourne, Victoria, Australia

7. Murdoch Children's Research Institute, Victoria, Australia

\section{Corresponding Author:}

Professor Sonia Grover

Department of Paediatric \& Adolescent Gynaecology

The Royal Children's Hospital

50 Flemington Road, Parkville, Victoria 3052

E: sonia.grover@rch.org.au

T: +619345 5513

F: +6193458666

This is the author manuscript accepted for publication and has undergone full peer review but has not been through the copyediting, typesetting, pagination and proofreading process, which may lead to differences between this version and the Version of Record. Please cite this article as doi: $10.1111 /$ jog.14532

This article is protected by copyright. All rights reserved. 
Running Title: Dysmenorrhoea in Young People

\section{Abstract}

Aim

To describe the characteristics, management, and outcomes of a cohort of young people with dysmenorrhea presenting to a tertiary adolescent gynaecology service, managed primarily with medical interventions.

Methods

A retrospective cohort study was conducted at the Royal Children's Hospital in Melbourne, Australia. Data were collected from medical records of patients presenting with dysmenorrhea and/or pelvic pain.

Results

Of 154 patients, mean age of presentation was 15.7 years $(S D=2.2)$ and mean duration of pain was 14.9 months (SD=10.8). Regular cycles were reported by $64.5 \%$, and heavy menstrual bleeding (HMB) in 67.8\%. Patients self-reporting HMB reported less pain on the day prior to menses than those not reporting HMB $(p<0.005)$. At follow-up, therapeutic interventions included nonsteroidal anti-inflammatory drugs, tranexamic acid, and cyclic or continuous combined oral contraceptive pills. Laparoscopies were undertaken in 12 (8.1\%) patients, with normal findings in 8 (66.7\%). Secondary dysmenorrhea was identified in 10 patients: of these, endometriosis was identified in one patient and unilateral obstructive Müllerian anomalies in six. Overall, $92.2 \%$ of patients had improvement in symptoms after treatment.

\section{Conclusion}

Laparoscopy and endometriosis rates in patients presenting to a tertiary centre were lower than previously reported, with most patients achieving symptom improvement without laparoscopy. 
Keywords: adolescent, dysmenorrhea, endometriosis, laparoscopy, pelvic pain, prostaglandin

\section{Definitions}

The World Health Organization defines adolescents and young people as those between the ages of 10 and 19, and 10 and 24 respectively. In our manuscript, we have included patients between the ages of 9 and 22, referred to as "young people."

\section{Introduction}

Dysmenorrhea is common in young people, with reported rates of 20 to $90 \% \cdot{ }^{1,2}$ It is classically described as either primary or secondary.

Primary dysmenorrhea is thought to occur in ovulatory cycles²: the fall in progestogens results in an inflammatory process which provokes endometrial shedding by way of the production of inflammatory mediators in the endometrial tissue. These inflammatory mediators incite a range of associated local and systemic symptoms that occur just prior to or during menstruation.1,2 Nonsteroidal anti-inflammatory drugs (NSAIDs) and the combined oral contraceptive pill (COCP) are recommended as first line treatments for patients presenting with primary dysmenorrhea and associated symptoms. ${ }^{1}$

Secondary dysmenorrhea, where underlying pathology is present, has been described in approximately $10 \%$ of young patients with dysmenorrhea; endometriosis is the most commonly identified underlying cause. ${ }^{2,3}$ Of those who have failed first line management with NSAIDs and COCP, $62 \%$ (range $25-100 \%$ ) have been found to have visually proven endometriosis at laparoscopy, with reported rates of up to $73.5 \%$ in patients with severe dysmenorrhea. ${ }^{4}$ 
Laparoscopy has thus been recommended as a means of identifying endometriosis in patients whose symptoms persist despite the use of NSAIDS and COCP beyond 3-6 months. ${ }^{2-7}$ Other causes of secondary dysmenorrhea in younger patients include Müllerian anomalies, ovarian masses, and pelvic inflammatory disease. ${ }^{2}$ Obstructed reproductive tract anomalies have been reported in three to eight percent of adolescents with dysmenorrhea presenting to specialist hospital clinics. ${ }^{6,8}$

Whilst laparoscopy is still considered the gold standard for the diagnosis of endometriosis, recommending the early use of laparoscopy in adolescents with dysmenorrhea raises significant concerns. Several studies have demonstrated a recurrence of pain within 12-18 months, with many young women then undergoing repeat surgical procedure(s). ${ }^{9,10}$ Predictors for repeat laparoscopy for pelvic pain include younger age and previous pelvic laparoscopy. ${ }^{11,12} \mathrm{~A}$ placebo effect of up to $30 \%$ has been reported in the literature, whereas up to $20 \%$ of women describe no improvement in symptoms. ${ }^{13}$ Medical treatment is proven to be effective for symptom control in adolescents with dysmenorrhea ${ }^{14-16}$ and recently has been shown to be associated with a reduction in size of endometriotic nodules in cases of moderate to severe rectovaginal endometriosis. ${ }^{17}$ Furthermore, there is a cohort of women who develop pain syndromes and myofascial pain following surgery. ${ }^{1,18}$ Finally, there is evidence that the pain sensitivity threshold of women with dysmenorrhea is lower than the pain sensitivity threshold of women without dysmenorrhea, regardless of the presence or absence of endometriosis. ${ }^{19}$

Our study therefore aims to clarify the characteristics of dysmenorrhea in young patients and to determine if adequate symptom control is achievable with a predominantly medical approach, with use of laparoscopy limited to patients with ongoing pain despite attempts to achieve complete suppression of menses.

\section{Methods}

This article is protected by copyright. All rights reserved. 
Patients presenting with dysmenorrhea to the Department of Paediatric and Adolescent Gynaecology at the Royal Children's Hospital (RCH) in Melbourne, Australia between 1997 and 2004 were identified from the outpatient record database. All patients had been referred by a primary care practitioner (family physician or paediatrician). Patients who were referred with pain but whose primary diagnosis was non-gynaecologic (for example: abdominal wall trigger points, irritable bowel syndrome, bladder pain syndrome), were excluded only when pelvic pain or dysmenorrhea was not a substantial component of their symptoms. However, patients in whom pelvic pain or dysmenorrhea was the chief symptom in their referral or presentation, even in the setting of other conditions, were still included. ICD codes for genital tract anomalies for the same period were also identified and cases where unilateral obstruction was present, but menstruation could occur from one functional side of the Müllerian system, were included. A retrospective chart review was performed and data extracted regarding age of menarche, age at onset of dysmenorrhea, age at presentation to our clinic, associated symptoms, pattern of menses, school missed due to dysmenorrhea, medications trialled prior to referral and those prescribed by the specialist adolescent gynaecologists, investigations prior to referral and during attendance at the clinic, operative procedures and findings, and outcomes at subsequent clinic appointments. All patients were seen in consultation by 1 of 3 consultant paediatric gynaecologists and/or 1 fellow under supervision. A standard approach to management of dysmenorrhea was employed (Figure 1). This was tailored to individual patient's medication contraindications and patient preferences at the discretion of the treating gynaecologist. Laparoscopies were performed by 1 of 3 consultant gynaecologists specializing in paediatric \& adolescent gynaecology. HMB was documented as self-reported heavy menstrual loss. A regular menstrual cycle was defined as 25 to 35 days.

Information regarding socioeconomic status was gained by utilizing the postcode related data available from the Australian Bureau of Statistics for Socio Economic Information for Area. ${ }^{20}$ 
All data analysis was performed using Stata 11. Chi Square, Fisher's exact and logistic regression were used to test correlation. The project was undertaken with $\mathrm{RCH}$ Human Research and Ethics approval (CA29080) as a clinical audit project and as such did not require patient consent.

\section{Results}

A total of 154 patients with dysmenorrhea or pelvic pain were identified, accounting for $9 \%$ of all outpatient visits in this time period. Age of presentation was between 9 and 22 years (mean 15.7, SD=2.2). Age at menarche was between 9 and 14 years (mean 11.8, SD=1.3) and mean interval from menarche to presentation was 3.8 years $(S D=1.8)$. Mean duration of pain was 14.9 months $(\mathrm{SD}=10.8)$.

School absenteeism was only recorded in a small subset of girls and therefore was not included in analysis.

An uneven distribution of socioeconomic background was found. Although the majority of patients fell within the middle socio-economic bracket, three-quarters were noted to reside in socio-economic areas above the $50^{\text {th }}$ percentile.

Self-assessment of menstrual flow was recorded in 143 patients: of these, $97(67.8 \%)$ reported HMB and 28 (19.5\%) reported the presence of clots in menstrual loss, although this was not further quantified. Other than presence of clots, there was no difference in baseline characteristics of patients reporting and not reporting HMB (Table 2).

The menstrual cycle pattern was recorded in 138 (89.6\%) patients. Of these, regular cycles were reported in 89 (64.5\%).

The exact timing and pattern of pain was noted in 126 (81.8\%) patients. In 84.9\%, the pain was most severe on days 1 and 2 of menses; 38.1\% reported pain on days 3 to 4 , and $15.1 \%$ on days 5 to 6 . Pain began the day prior to menses in $54.7 \%$ and between two and seven days before menses in $23.8 \%$.

This article is protected by copyright. All rights reserved. 
Patients reporting HMB $(n=97)$ experienced significantly less pain on the day prior to menses than those without HMB $(n=28)(p<0.005)$. There was no association between duration of menses and $\mathrm{HMB}$, and no significant difference in pain pattern between girls reporting regular cycles and those reporting irregular cycles. (Table 2) Associated symptoms were recorded in 148 patients: nausea (39.9\%), diarrhoea (33.1\%), headaches (32.4\%), tiredness (28.4\%), back pain (27.7\%), vomiting (26.4\%), irritable mood (14.9\%), syncope (12.8\%), anorexia (10.1\%), dizziness (8.8\%), constipation (7.4\%) and bloating (4.7\%). Eleven (7.4\%) patients did not report any associated symptoms. (Table 1) There was a significant correlation between nausea and other symptoms: vomiting $(p<0.005)$, diarrhoea $(p<0.05)$, headaches $(p<0.005)$ and tiredness $(p<0.01)$. Overall, 70.8\% reported nausea, vomiting, diarrhoea, dizziness or feeling faint, with $29.8 \%$ reporting 3 or more of these symptoms. Of the patients who did not report any of this cluster of symptoms, $74.4 \%$ had HMB.

\section{Medical Management}

A range of pain management approaches had been trialled by most patients prior to referral: NSAIDs (71.4\%), cyclic COCP (23.1\%), alternative therapies (8.2\%), continuous COCP (5.4\%), oral progestogens (4.8\%), tranexamic acid $(0.7 \%)$ and depot medroxyprogesterone acetate (DMPA) (0.7\%). Twenty patients (13.6\%) reported no medications or interventions prior to their specialist consultation.

During the first consultation, patients were prescribed or given instructions for optimal use of NSAIDs (51.4\%), cyclic COCPs (26.4\%), continuous COCPs (20.8\%), tranexamic acid (11.5\%), alternative medicine (6.8\%) and/or oral progestogens (2.7\%).

During the course of the subsequent consultations, NSAIDs were prescribed for $60.1 \%$, continuous COCPs for $49.3 \%$ and cyclic OCPs for $46.6 \%$. Other management, used in combination or alone, included tranexamic acid (20.7\%), oral progestogens (12.6\%), alternative 
treatment (8.1\%), DMPA (4.5\%), etonorgestrel implant (1.8\%) and levonorgestrel intrauterine system (IUS) (1.8\%).

\section{$\underline{\text { Surgical management }}$}

\section{Laparoscopy}

Four patients had undergone laparoscopy prior to referral to our clinic: three were reportedly normal and one had findings of mild endometriosis. This patient had not trialled any medical treatments pre-operatively. Twelve patients (8.1\%) underwent laparoscopy for further investigation, including one patient who underwent repeat laparoscopy. Indication for laparoscopy was $\geq 12$ months of persistent pain despite trial of $\geq$ two hormonal medications (Figure 1). Eight of these patients had ongoing pain despite the use of continuous COCP. Abnormal findings at laparoscopy were noted in four patients: presence of minor adhesions in two patients, ovarian cyst in one patient and histologically proven mild endometriosis in one patient. No pelvic pathology was found in the remaining eight cases. The patient who was found to have mild endometriosis had not achieved menstrual suppression despite medical management, including use of $\mathrm{GnRH}$-analogue.

\section{Correction of anomalies}

Six patients (3.3\%) were identified with uterine didelphys, obstructed hemivagina with ipsilateral renal agenesis. All were managed operatively with excision of the vaginal septum. None of these patients underwent laparoscopy. The mean age at presentation of this group was 14.2 years $(S D=2.5)$. All had resolution of pain following correction of the anomaly.

\section{Follow-up}

This article is protected by copyright. All rights reserved. 
Of the 154 patients in the study, 38 (24.8\%) did not return for clinical follow-up. Of these, 20 had not used any interventions or medications prior to their first consultation.

Of the 115 (74.7\%) patients who presented for follow-up, median follow-up was 11 months (IQR: 3-26). Median and mean number of clinic visits were 3 and $3.9(S D=3.52)$ respectively. Symptoms were reported to be resolved or well-controlled following medical/surgical intervention by $59.7 \%$ of patients, while $32.2 \%$ were somewhat improved. These were subjective measures documented at follow-up visits. Thirteen patients (8.4\%) reported ongoing dysmenorrhea. (Figure 2)

\section{Discussion}

This study population represents a group of young patients with dysmenorrhea referred by primary care practitioners, paediatricians and other general gynaecologists to a specialised paediatric and adolescent gynaecology clinic at a tertiary paediatric hospital. The cohort in this study represents patients referred with pelvic pain or dysmenorrhea, but excludes those who were found to have alternative non-gynaecological causes for their pelvic or abdominal-pelvic pain such as abdominal wall trigger points, irritable bowel syndrome or bladder pain syndrome. The mean age of presentation and of menarche of our population is similar to other reports of young patients with dysmenorrhea. ${ }^{21-23}$ Although some studies have suggested that early menarche is a risk factor for dysmenorrhea ${ }^{1}$, this is not supported by our findings. Reports have previously suggested that patients with higher socioeconomic status are at higher risk of dysmenorrhea. ${ }^{24}$ Our study is congruent with this observation; however, we hypothesize that this may simply reflect improved access to medical services and/or improved health literacy. Primary dysmenorrhea has been reported to occur with ovulatory cycles, rather than immediately after menarche, due to an increase in prostaglandin production triggered by a fall in progestogens. ${ }^{1}$ More recently, this theory has come into question secondary to a lack of difference in dysmenorrhea rates between ovulating and non-ovulating patients. ${ }^{25}$ One third of 
our adolescent dysmenorrheic patients reported irregular cycles, with anovulation likely to be the cause, further challenging the link between ovulation and the subsequent fall in progestogens as the key trigger to prostaglandin production. The symptom cluster of nausea, vomiting, diarrhoea, headaches and dizziness, found in $70.8 \%$ of our study cohort, has been reported by others to be associated with primary or prostaglandin-induced dysmenorrhea ${ }^{1}$, implying that prostaglandin release and the inflammatory process of menstrual shedding are still present.

Reports on the association between HMB and dysmenorrhea are mixed, with rates of $10.8 \%$ to $85.6 \%{ }^{21,26}$ As the reliability of self-reports of menstrual loss is questionable, particularly in this young population ${ }^{27}$, and no standardized bleeding assessment tool was used, the significance of this finding is unclear. Nevertheless, our data suggest the possibility of a different pain pattern in girls self-reporting HMB, as they appear to have less systemic prostaglandinassociated symptoms and less pain on the days prior to menses when compared to those who did not report HMB. Approaches aimed at reducing menstrual blood loss, such as tranexamic acid, may be useful in this sub-group. Further studies are required to clarify this possible bimodal pattern.

It has been recommended that laparoscopy be undertaken for investigation of dysmenorrhea where NSAIDs and the COCP have failed. ${ }^{7}$ In fact, studies on dysmenorrhea in adolescents have tended to specifically report on findings in this population ${ }^{6,23,28}$, rather than on a cohort presenting with dysmenorrhea or pain. The total laparoscopy rate in our study (including four patients in whom laparoscopy was undertaken prior to referral to our service) was only $10.4 \%$. It is our routine practice to aim for complete menstrual suppression for six months prior to laparoscopy for dysmenorrhea. We hypothesize that cyclic use of the COCP with subsequent endometrial shedding results in the release of prostaglandins and inflammatory cytokines during the hormone-free-interval, permitting the propagation of pain induced by these substances. 
Our routine use of continuous COCP may thus account for both the low laparoscopy rate and the low endometriosis rate found in our population. Of the patients who had persistent symptoms despite medical management, only one was found to have endometriosis; menstrual suppression had not been successfully achieved in this patient. Overall, $92.2 \%$ of our cohort had self-reported improved or resolved symptoms despite a very low surgical intervention rate. Six (3.3\%) of our patients had a hemi-obstructed congenital anomaly. These patients had resolution of symptoms following primary corrective surgery without the addition of laparoscopy. Overall, $10(6.5 \%)$ of our patients were found to have a secondary cause of dysmenorrhea. This is much lower than that reported by other studies and may partly reflect our low laparoscopy rate and possible undiscovered secondary causes. Additionally, the rate of positive findings (33.3\%) amongst those who did undergo laparoscopy is lower than reported by others. However, the 13 patients who reported their symptoms had not improved or were not well controlled despite receiving medical management may increase this number were they to undergo laparoscopy.

We acknowledge that there are several limitations to our study. Firstly, our inclusion and exclusion criteria resulted in a population of patients whose symptoms were predominately limited to dysmenorrhea and/or pelvic pain without the presence of other pain syndromes. Thus, the results cannot be extrapolated to patients with these co-morbid conditions. Equally, our findings may be valuable to those patients presenting with chiefly isolated dysmenorrhoea or pelvic pain. Additionally, 38 (24.8\%) of our patients did not attend follow-up after their first visit to our clinic. Of these were 20 who had not undertaken any interventions prior to their first consultation and thus the information given and simple advice may have resolved their concerns. It is possible that the remaining 18 patients had improvement in their symptoms after initial medical management, and therefore did not feel the need to return, or alternatively were dissatisfied with initial treatment and thus sought further management elsewhere. Finally, several of our measures, including presence of HMB and degree of improvement in symptoms, 
were subjective and descriptive, thus obtaining quantitative results was not possible in these instances.

\section{Conclusion}

In our study, most patients were successfully treated with medical management without the need for surgical intervention, and we found a smaller proportion of patients with secondary dysmenorrhea from obstructive anomalies and endometriosis than previously reported. We propose that in patients presenting with dysmenorrhea and/or pelvic pain, complete menstrual suppression be included in a trial of medical therapy prior to moving onto to considering surgical investigation. The long-term follow-up of our cohort has been reported in a separate study and sheds further encouraging, positive light on this conservative approach to dysmenorrhea in young patients. ${ }^{14}$

Acknowledgements: none

Disclosure: None of the authors had a conflict of interest or received any financial support for this project.

\section{References}

1. lacovides S, Avidon I, Baker FC (2015) What we know about primary dysmenorrhea today: a critical review. Hum Reprod Update 21:762-778

2. Harel Z (2006) Dysmenorrhea in adolescents and young adults: etiology and management. J Pediatr Adolesc Gynecol 19:363-371

This article is protected by copyright. All rights reserved. 
3. Janssen EB, Rijkers AC, Hoppenbrouwers K, Meuleman C, D'Hooghe TM (2013)

Prevalence of endometriosis diagnosed by laparoscopy in adolescents with dysmenorrhea or chronic pelvic pain: a systematic review. Hum Reprod Update 19:570582

4. Dowlut-McElroy T, Strickland JL (2017) Endometriosis in adolescents. Curr Opin Obstet Gynecol 29:306-309

5. Sanfilippo j, Erb T (2008) Evaluation and management of dysmenorrhea in adolescents. Clin Obstet Gynecol 51:257-267

6. Vercellini P, Fedele L, Arcaini L, Bianchi S, Rognoni MT, Candiani GB (1989) Laparoscopy in the diagnosis of chronic pelvic pain in adolescent women. J Reprod Med 34:827-830

7. ACOG Committee Opinion (2005) ACOG Committee Opinion. Number 310, April 2005. Endometriosis in adolescents. Obstet Gynecol 105:921-927

8. Rossier M, Bays V, Vial Y, Achtari C (2008) [Congenital uterine anomalies: diagnosis, prognosis and management in 2008]. Rev Med Suisse 4:2253-2254, 2256-2258, 2260 passim

9. Koga K, Takamura M, Fujii T, Osuga Y (2015) Prevention of the recurrence of symptom and lesions after conservative surgery for endometriosis. Fertil Steril 104:793-801

This article is protected by copyright. All rights reserved. 
10. Jarrell J (2008) Diagnostic and operative laparoscopy in Alberta 1994-2006. J Obstet Gynaecol Can 30:1045-1049

11. Cheong Y, Tay P, Luk F, Gan HC, Li TC, Cooke I (2008) Laparoscopic surgery for endometriosis: How often do we need to re-operate? J Obstet Gynaecol 28:82-85

12. Mirowska-Allen K (2016) Chronic pelvic pain- factors associated with a surgical intervention. MD, University of Melbourne, Melbourne, Australia

13. Abbott J, Hawe J, Hunter D, Holmes M, Finn P, Garry R (2004) Laparoscopic excision of endometriosis: a randomized, placebo-controlled trial. Fertil Steril 82:878-884

14. Knox, B., Y. Ong, M. Bakar and S. Grover (2019). "A longitudinal study of adolescents dysmenorrhoea into adulthood." Eur J Pediatr 178: 1325-1332.

15. J.C. Robinson, S. Plichata, C.S. Weisman, et al. Dysmenorrhea and use of oral contraceptives in adolescent women attending a family planning clinic. Am J Obstet Gynecol, 166 (1992), p. 578

16. A.R. Davis, C. Westhoff, K. O'Connell, et al. Oral contraceptives for dysmenorrhea in adolescent girls: A randomized trial. Obstet Gynecol, 106 (2005), p. 97

17. Vercellini P, Pietropaolo G, De Giorgi O, Pasin R, Chiodini A, Crosignani PG (2005) Treatment of symptomatic rectovaginal endometriosis with an estrogen-progestogen combination versus low-dose norethindrone acetate. Fertil Steril 84:1375-1387

This article is protected by copyright. All rights reserved. 
18. Jarrell J (2010) Myofascial pain in the adolescent. Curr Opin Obstet Gynecol 22:393398

19. As-Sanie S, Harris RE, Harte SE, Tu FF, Neshewat G, Clauw DJ (2013) Increased pressure pain sensitivity in women with chronic pelvic pain. Obstet Gynecol 122:10471055

20. Australian Bureau of Statistics (2006) http://www.abs.gov.au/AUSSTATS/abs@.nsf/allprimarymainfeatures/356A4186CCDDC 4D1CA257B3B001AC22C?opendocument.

21. Parker M, Sneddon A, Arbon P (2010) The menstrual disorder of teenagers (MDOT) study: determining typical menstrual patterns and menstrual disturbance in a large population-based study of Australian teenagers. BJOG 117:185-192

22. Ortiz M, Rangel-Flores E, Carrillo-Alarcon L, Veras-Godoy H (2009) Prevalence and impact of primary dysmenorrhea among Mexican high school students. Int J Gynaecol Obstet 107:240-243

23. Dun EC, Kho KA, Morozov VV, Kearney S, Zurawin JL, Nezhat CH (2015) Endometriosis in adolescents. JSLS 19

24. Klein J, Litt I (1981) Epidemiology of adolescent dysmenorrhea. Pediatrics 68:661-664

This article is protected by copyright. All rights reserved. 
25. Espin Lopez L, Carrillo Verdejo E, Gonzalez Javier F, Ordonana Martin J, Gomez-Amor J (2010) Incidence of anovulatory menstrual cycles among dysmenorrheic and nondysmenorrheic women: effects on symptomatology and mood. Psicothema 22:654-658

26. El-Gilany A, Badawi K, El-Fedawy S (2005) Epidemiology of dysmenorrhoea among adolescent students in Mansoura, Egypt. East Mediterr Health J 11:155-163

27. Fraser IS, McCarron G, Markham R (1984) A preliminary study of factors influencing perception of menstrual blood loss volume. Am J Obstet Gynecol 149:788-793

28. Laufer MR, Goitein L, Bush M, Cramer DW, Emans SJ (1997) Prevalence of endometriosis in adolescent girls with chronic pelvic pain not responding to conventional therapy. J Pediatr Adolesc Gynecol 10:199-20

\section{Legends for Tables \& Figures}

Table 1. Patient descriptors and associated symptoms

This article is protected by copyright. All rights reserved. 
Table 2. Characteristics of patients reporting or not reporting HMB at presentation

Figure 1. Royal Children's Hospital Paediatric \& Adolescent Gynaecology protocol for management of dysmenorrhoea. a. offered to all patients with dysmenorrhoea, $b$. offered to all patients with HMB. c. oral, intramuscular, subdermal, or intrauterine.

Figure 2. Symptom control at the time of follow-up

This article is protected by copyright. All rights reserved. 


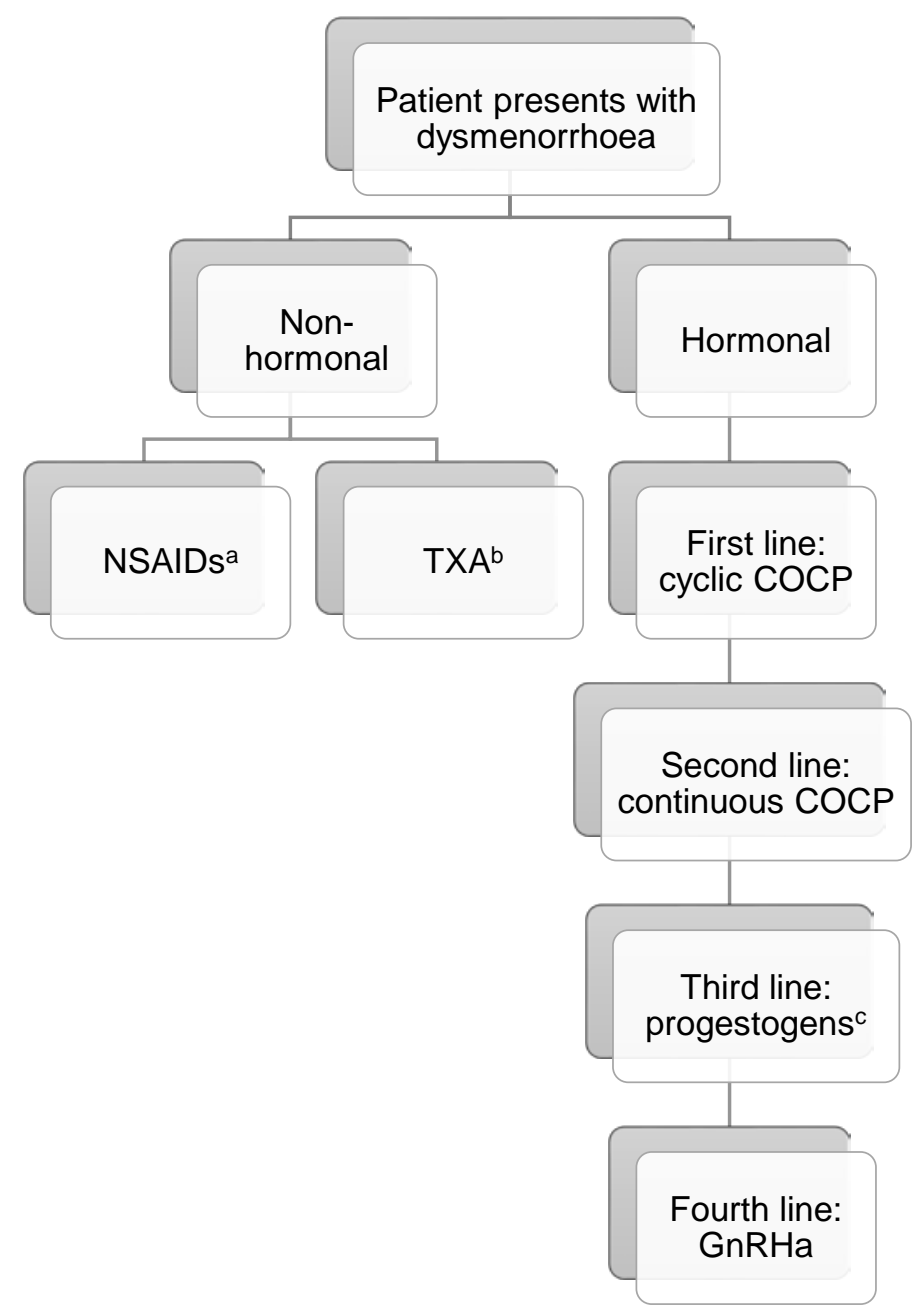

Figure 1. Royal Children's Hospital Paediatric \& Adolescent Gynaecology protocol for management of dysmenorrhoea. a. offered to all patients with dysmenorrhoea, b. offered to all patients with HMB. c. oral, intramuscular, subdermal, or intrauterine. 


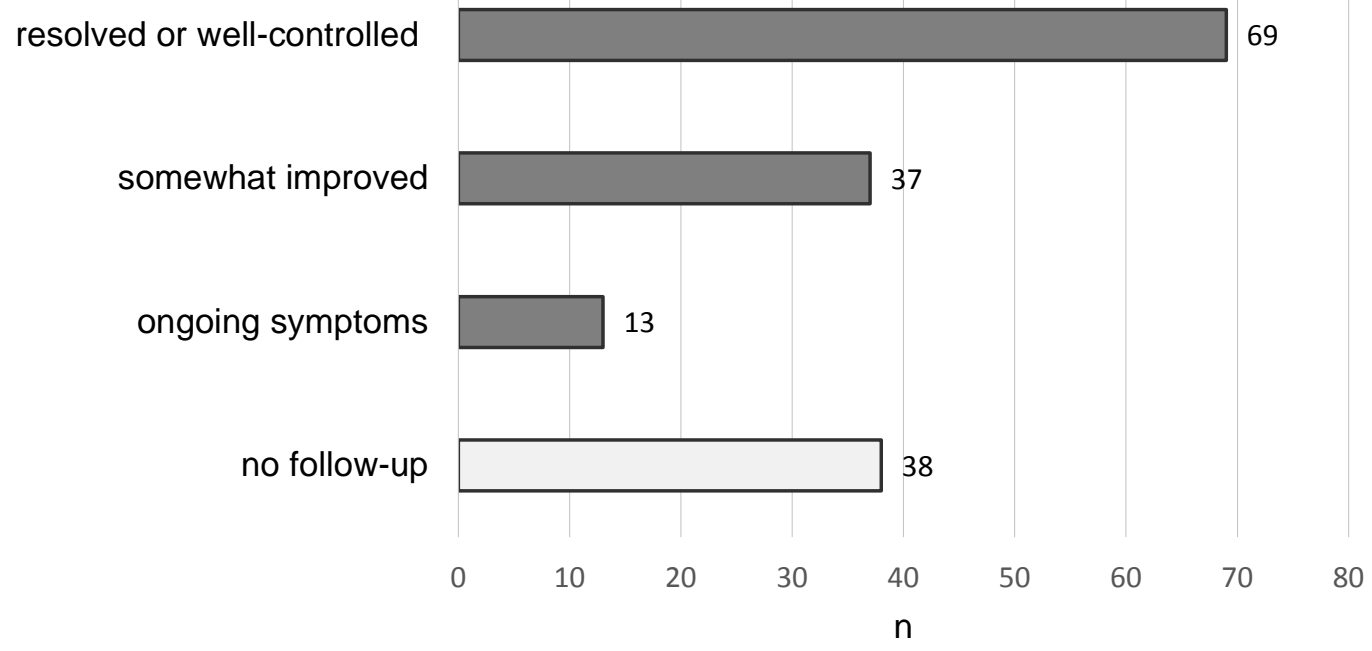

Figure 2. Symptom control at the time of follow-up

This article is protected by copyright. All rights reserved. 


\begin{tabular}{ll} 
Demographics $(\mathrm{N}=154)$ & Mean $(\mathrm{SD})$ \\
\hline Age of presentation $(\mathrm{y})$ & $15.7(2.2)$ \\
Age of menarche $(\mathrm{y})$ & $11.8(1.4)$ \\
Time from menarche to presentation $(\mathrm{y})$ & $3.8(1.8)$ \\
Duration of pain $(\mathrm{m})$ & $14.9(10.8)$ \\
& \\
Cycle regularity $(\mathrm{N}=138)$ & $\mathrm{n}(\%)$ \\
\hline Regular $(25-35 \mathrm{~d})$ & $89(64.5)$ \\
Irregular & $49(35.5)$ \\
& \\
Menstrual flow $(\mathrm{N}=143)$ & $\mathrm{n}(\%)$ \\
\hline HMB & $97(67.8)$ \\
Clots in menstrual loss & $28(19.5)$ \\
& \\
Associated symptoms $(\mathrm{N}=148)$ & $\mathrm{n}(\%)$ \\
\hline Nausea & $59(39.9)$ \\
Diarrhoea & $49(33.1)$ \\
Headache & $48(32.4)$ \\
Tiredness & $42(28.4)$ \\
Back pain & $41(27.7)$ \\
Vomiting & $39(26.4)$ \\
Irritable mood & $22(14.9)$ \\
Syncope & $19(12.8)$ \\
Anorexia & $15(10.1)$ \\
Dizziness & $13(8.8)$ \\
Constipation & $11(7.4)$ \\
Bloating & $7(4.7)$ \\
No associated symptoms & $11(7.4)$ \\
\end{tabular}

Table 1. Patient descriptors and associated symptoms

This article is protected by copyright. All rights reserved. 


\begin{tabular}{llll} 
& $\begin{array}{l}\text { no HMB } \\
(\mathrm{n}=46)\end{array}$ & $\begin{array}{l}\mathrm{HMB} \\
(\mathrm{n}=97)\end{array}$ & \\
& mean (SD) & mean (SD) & p-value \\
\hline $\begin{array}{l}\text { age of presentation } \\
\text { age of menarche }\end{array}$ & $15.8(2.4)$ & $15.7(2.1)$ & 0.84 \\
& $11.8(1.1)$ & $11.7(1.4)$ & 0.74 \\
& $\mathrm{n}(\%)$ & $\mathrm{n}(\%)$ & $\mathrm{p}$-value \\
\hline regular cycles $(5)$ & $27(61.4)$ & $62(66.0)$ & 0.6 \\
alternate medications & $5(10.9)$ & $7(7.2)$ & 0.46 \\
NSAIDs & $35(76.1)$ & $67(69.1)$ & 0.39 \\
TXA & $0(0.0)$ & $1(1.0)$ & 0.49 \\
progesterone & $1(2.2)$ & $6(6.2)$ & 0.3 \\
DMPA & $0(0)$ & $1(1.0)$ & 0.49 \\
cyclic OCP & $10(21.7)$ & $22(22.7)$ & 0.9 \\
continuous OCP & $2(4.4)$ & $6(6.2)$ & 0.66 \\
etonorgestrel implant & $0(0)$ & $0(0)$ & 1 \\
levonorgestrel IUD & $0(0)$ & $0(0)$ & 1 \\
clots & $1(2.2)$ & $29(29.9)$ & $<0.01$
\end{tabular}

Table 2. Characteristics of patients reporting or not reporting HMB at presentation 


\section{University Library}

\section{- M M N E R VA A gateway to Melbourne's research publications}

Minerva Access is the Institutional Repository of The University of Melbourne

Author/s:

Sachedina, A;ABU Bakar, M;Dunford, AM;Morris, A;NUR Azurah, AG;Grover, SR

Title:

Dysmenorrhea in young people: Experiences from a tertiary center with a focus on conservative management

Date:

2020-10-20

Citation:

Sachedina, A., ABU Bakar, M., Dunford, A. M., Morris, A., NUR Azurah, A. G. \& Grover, S. R. (2020). Dysmenorrhea in young people: Experiences from a tertiary center with a focus on conservative management. JOURNAL OF OBSTETRICS AND GYNAECOLOGY RESEARCH, 47 (1), pp.352-358. https://doi.org/10.1111/jog. 14532.

Persistent Link:

http://hdl.handle.net/11343/276463 